\title{
Sobre la necesidad de desconectar: algunos datos y propuestas
}

\section{On the need to unplug: some facts and proposals}

\author{
Concepción Naval ${ }^{1}$, Javier Serrano-Puche ${ }^{2}$, Charo Sádaba ², Elena Arbués
}

Universidad de Navarra, Facultad de Educación y Psicología, Pamplona, Navarra, España. \{cnaval, earbues\}@unav.es

2 Universidad de Navarra, Facultad de Comunicación, Pamplona, Navarra, España. \{jserrano, csadaba\}@unav.es

\begin{abstract}
Resumen
Si algo caracteriza la sociedad de la información en la que vivimos es la invasión tecnológica y la necesidad de conectividad permanente, especialmente entre los jóvenes. Estos y otros rasgos configuran la era de la hiperconectividad (Reig y Villchez, 2013). Ante esta realidad hay quien advierte entre los usuarios cierta fatiga tecnológica, por lo que, desde diversos ámbitos, se empieza a considerar la necesidad de repensar la aproximación tecnología, de recapacitar sobre los efectos que la tecnológica tiene en nuestras vidas. Su apuesta es la desconexión digital, propuesta que puede enmarcarse dentro de una corriente más amplia, el movimiento slow, que aboga por un estilo de vida más pausado (Honoré, 2004). No se trata de eliminar por completo la vida online, sino de instaurar períodos regulares de desconexión digital para cultivar la comunicación cara a cara; abogando por el desarrollo de un ocio que, en parte, prescinda de las tecnologías digitales y por una recuperación de las fronteras entre el tiempo laboral y el de descanso (Wajcman et al., 2009)
\end{abstract}

En este trabajo ahondamos en la literatura académica y divulgativa que aborda esta cuestión y nos referimos a diversas investigaciones, tanto teóricas como empíricas.

Desde un punto de vista educativo señalamos cómo, en la actualidad, la educación mediática y el impulso de la competencia digital es una prioridad de los sistemas educativos y de los principales organismos internacionales (Kubey, 2003; Martens 2010). Es fundamental que los ciudadanos aprendan los nuevos lenguajes, adquieran la capacidad de discernir y de valorar críticamente la gran cantidad de medios e información disponible y realicen un uso seguro de ellos. Quizá convenga considerar, como un componente sustancial de la competencia digital, el aprender a desconectar, para conectar de otro modo.

\section{Palabras Clave}

Conectividad; Desconexión Digital; Movimiento Slow; Educación Mediática; Competencia Digital; Desconexión.

\begin{abstract}
If there is one point that stands out in our information society it is that we are in the midst of a technological invasion and that we, and particularly young people, feel the need to be permanently connected. These and other traits are what give shape to the era of hyperconnectivity (Reig y Villchez, 2013). Regarding this reality, there are those who see a certain technological fatigue amongst users, and so, from certain perspectives, there is a feeling that we need to rethink the technological approach, to reconsider the effects that technology has on our lives.
\end{abstract}

The suggestion is digital unplugging, a proposal which can be included in a broader movement, the Slow Movement, which champions a more unhurried lifestyle (Honoré, 2004). This does not mean eliminating online life completely, but rather establishing regular periods of digital time-out in order to develop face-to-face communication; they suggest cultivating leisure periods which, to a certain extent, would dispense with digital technology and would reconstruct the borders between the time for work and the time for rest (Wajcman et al., 2009)

In this work we make an in-depth study of the academic and informative literature which deals with this issue and we refer to varied research, both technical and empirical. From an education perspective we point out how, at present, media education and the promotion of digital competence is a priority in the systems of education and of the main international bodies (Kubey, 2003; Martens 2010). It is absolutely fundamental that people learn these new languages, acquire the capacity to critically differentiate and evaluate the enormous amount of media and sources of information which is available, and use this safely. But perhaps one important component of digital competence should be learning how to disconnect, in order to make connections in another way.

\section{Keywords}

Connectivity; Digital Disconnection; Slow Movement; Media Literacy; Digital Competence; Unplugging. 


\section{Competencia digital y educación mediática}

La sociedad de la información y de la comunicación se caracteriza por la posibilidad de acceder, almacenar y transmitir información, generar conocimiento y seguir aprendiendo a lo largo de toda la vida (Sancho, 2001). Vivimos en un mundo en el que las tecnologías de la información y de la comunicación (TIC) ejercen una considerable influencia sobre la manera de organizar nuestra vida, de comportarnos, sobre los hábitos de vida y, en general, sobre nuestra manera de ver y comprender el mundo (Pavón, 2005). Las TIC están cada vez más presentes en todos los ámbitos: trabajo, ocio, diversión, información, participación, aprendizaje, relaciones sociales, formas de comunicarse, etc. De forma que es posible alcanzar una situación de conectividad permanente y empieza a ser usual la expresión "vida digital". Las redes sociales son una de las aplicaciones más populares de internet. Se entienden como una forma de interacción social donde se produce un intercambio interpersonal logrando un sentido de integración. En ellas es posible mantener contactos laborales, entre amigos, reencuentros, etc. Pero también son un lugar para compartir información, conocimientos y que posibilitan la participación.

Este hecho, desde el punto de vista educativo, sugiere considerar e incidir en determinados aspectos, proporcionando una adecuada educación mediática. Un debate abierto en la comunidad educativa es por qué educar para el uso de las tecnologías de la información y de la comunicación, y sobre cómo y quién lleva a cabo este cometido (Campuzano, 1992; Sancho, 2001; Potter, 2010; Naval y Arbués, $2012,2013,2014)$. Es fundamental que los ciudadanos aprendan los nuevos lenguajes y adquieran la capacidad de discernir y valorar críticamente la gran cantidad de información disponible. No cabe duda de la importancia que esta área tiene tanto en el ámbito familiar, como en el de los medios de comunicación, y por supuesto en el marco de la educación formal.

En este sentido la alfabetización mediática juega un papel importante para la difusión de los conocimientos científicos en los medios de comunicación (Rosenbaum et al., 2008). Y lo hace precisamente en una sociedad en la que conviven diversos grupos sociales con rasgos culturales propios. Sin embargo, en relación a la tecnología, todos los grupos parecen compartir hábitos y herramientas. Podríamos decir que la cultura tecnológica es parte de la cultura de cualquier grupo social (Pavón, 2005), por lo que los medios tecnológicos se convertirían en medios que favorecen la comunicación entre culturas y la participación social.

Si bien la educación mediática y digital es una realidad que prospera con cierta dificultad, lo cierto es que en la actualidad es una prioridad tanto de los sistemas educativos como de los principales organismos internacionales. Según Kubey $(2003,352)$, "a worldwide movement in media literacy 
education has been growing for roughly twenty-five years now and has been marked by a number of recent developments". Sin duda los sistemas educativos nacionales europeos se han hecho eco de esta realidad y procuran proporcionar una adecuada educación en los medios a sus ciudadanos.

Los educadores que se ocupan de estos temas comparten en gran medida currículos, investigación y estrategias, y la competencia mediática es un objetivo educativo cada vez más presente en distintos países (Martens, 2010); sin embargo, el concepto media literacy sigue siendo una cuestión debatida y controvertida (Potter, 2010). Los currículums formulados en términos de competencias se refieren a este aspecto de la educación como competencia mediática y audiovisual, sin embargo en las últimas décadas se han utilizado diversos términos.

Según las revisiones de Bawden (2008) y de Gallado (2013) alfabetización informática era el término usado en los años 80 y se refería al nivel de experiencia y de familiaridad con los ordenadores y las aplicaciones informáticas (Hawkins y Paris, 1997).

En los años 90 ganó popularidad el término alfabetización informacional. Es un término enraizado en las disciplinas académicas de Biblioteconomía y Ciencias de la Información. Bundy (2004) define la alfabetización informacional como un set de habilidades que permiten a los individuos reconocer cuándo la información es necesaria y tener la capacidad de localizarla, evaluarla y utilizarla eficazmente.

El término alfabetización digital fue introducido por Gilster (1997) y lo define como la capacidad de entender y usar la información en múltiples formatos desde una amplia gama de fuentes cuando se presenta utilizando ordenadores. Este término implica algo más que la mera capacidad de utilizar software u operar con un dispositivo digital; representa la capacidad de una persona de realizar tareas con eficacia en un entorno digital.

En 2007 el Consejo y el Parlamento Europeo establecieron el marco de referencia europeo sobre las competencias clave para el aprendizaje permanente. Entre ellas se encuentra la denominada competencia digital, que se define como la competencia que "entraña el uso seguro y crítico de las tecnologías de la sociedad de la información para el trabajo, el ocio y la comunicación" (Comisión Europea, 2007, 7). Se indica que las capacidades necesarias son: buscar, obtener y tratar información; utilizarla de manera crítica y sistemática; utilizar herramientas para producir, presentar y comprender información compleja; utilizar la tecnología en el apoyo del pensamiento crítico, la creatividad y la innovación. Esta competencia requiere una actitud crítica y reflexiva, y se sustenta también en el interés por participar en comunidades y redes con fines culturales, sociales o profesionales.

Otro término relacionado con la competencia digital, aunque más amplio, es el de alfabetización mediática. Para Pérez-Tornero y Martínez (2011) ambos conceptos expresan la importancia que 
tiene la adquisición de nuevas competencias y habilidades en relación con las TIC y los medios. La alfabetización mediática se integra en el marco más amplio de la transformación cultural donde convergen medios electrónicos y digitales.

En el documento Media and Information literacy curriculum for teachers (Wilson et al., 2011), la alfabetización mediática e informacional se entiende como la disciplina que pretende dotar a los ciudadanos de los conocimientos básicos sobre el papel de los medios de comunicación y los dispositivos de información en las sociedades democráticas. Abarca un conjunto de competencias y conocimientos esenciales para el ciudadano del siglo XXI: posibilidad de participar en el sistema mediático, desarrollar su espíritu crítico y adquirir conocimientos a lo largo de la vida para participar en el desarrollo de la sociedad y convertirse en ciudadanos activos. En dicho documento se concibe la alfabetización mediática de manera holística, se destaca la importancia de que los profesores se alfabeticen en medios e información como estrategia para alcanzar un efecto multiplicador. Y se señalan las 7 competencias requeridas por los profesores para considerar que están alfabetizados mediáticamente. Pueden dar luz para entender cuáles son los cometidos de la competencia digital.

Son las siguientes:

- Entender el papel de los medios y de la información en la democracia.

- Comprender el contenido de los medios y sus usos.

- Acceder a la información de una manera eficaz y eficiente.

- Evaluar críticamente la información y las fuentes de información.

- Aplicar los formatos nuevos y los tradicionales en los medios.

- Situar el contexto sociocultural del contenido de los medios.

- Promover la alfabetización mediática e informacional entre los estudiantes y manejar los cambios requeridos.

En definitiva podríamos decir que Educación mediática es el término aconsejado por el Parlamento Europeo para referirse a la alfabetización mediática. En 2008, la Eurocámara planteó la necesidad de que la educación mediática formara parte de los planes de estudio, en todos los niveles de educación escolar, introduciendo para ello una asignatura de "Educación mediática" en los colegios (Informe del Parlamento Europeo 2008/2129 (INI)). Si algo podemos destacar en la evolución que este ámbito de la educación ha experimentado, y que hemos tratado de mostrar, es que se ha pasado de procurar, inicialmente, la familiaridad y experiencia con la tecnología para localizar y manejar información; a buscar, en la actualidad, que los ciudadanos comprendan el papel y usos de los medios utilizándolos 
de manera segura, crítica y reflexiva.

A tenor del vertiginoso avance de la innovación tecnológica y del crecimiento exponencial de su uso entre los jóvenes, no parece equivocado vaticinar que la educación mediática será un ámbito educativo sujeto a revisión y actualización. Considerando además que, hoy en día, la red se entiende como un espacio de socialización, de forma que la hiperconexión es uno de los soportes esenciales de las formas de comunicación e interacción social (Cáceres y Morales, 2014).

Así las cosas, en las últimas décadas hemos sido testigos de algunos fenómenos sociales realmente interesantes. Nos referimos concretamente a la explosión de las redes sociales y últimamente al fenómeno del unplugging. Curiosamente al hablar de competencia digital no se incluye habitualmente algo que acaso convendría considerar también: aprender a desconectar, para conectar de otro modo. De este fenómeno pasamos a tratar a continuación.

\section{Una brecha en la cultura de la hiperconectividad: el unplugging como fenómeno emergente}

La aparición y consolidación de internet en la vida cotidiana de las personas ha marcado un antes y un después en las prácticas comunicativas y en la interacción social (Jordan, 2013). Esta novedad propiciada por la red, como apuntan Rainie y Wellman (2012), forma parte de una "triple revolución" que pivota en torno a las redes sociales (entendidas en un sentido amplio), internet y los dispositivos móviles. Según estos autores, vivimos en una época de individuos interconectados a través de redes -el "nuevo sistema operativo social"-, que tienen en internet su plataforma de contacto e intercambio de información y a la que ya pueden acceder de manera constante y ubicua, gracias a la comunicación móvil.

La omnipresencia y ubicuidad de los dispositivos digitales no es, sin embargo, una mera cuestión cuantitativa, puesto que "su amplia difusión, personalización y la posibilidad de conexión permanente que crean, contribuyen a reconfigurar numerosos aspectos de la vida cotidiana, así como de los procesos de subjetivación y socialización contemporáneos" (Lasén, 2014, 7). En ese mismo sentido, como apunta Lipovetsky, "la red de las pantallas ha transformado nuestra forma de vivir, nuestra relación con la información, con el espacio-tiempo, con el consumo" $(2006,271)$.

En efecto, las tecnologías digitales han favorecido una flexibilidad cada vez mayor en las relaciones entre individuos y grupos, dando pie al desarrollo de lo que los autores denominan un "individualismo interconectado" (Wellman et al., 2003) o un "yo en red" (Papacharissi, 2011). Así, en paralelo a las relaciones tradicionales de pertenencia, proliferan las relaciones reticulares transitorias de alcance más limitado, caracterizadas por una menor rigidez y un mayor dinamismo (Pisani y Piotet, 2009). 
Aunque en gran medida depende de la actitud de cada persona, cabe constatar que, por sus propias características, las interacciones digitales no tienden de por sí a proporcionar un estilo de interacción que requiera un compromiso fuerte por parte de la persona, sino una comunicación más bien efímera, fragmentaria, cambiante. En ese sentido, el entorno digital le permite al usuario estar en compañía, pero preservando su individualidad (Turkle, 2011); ofreciendo, en definitiva, un modo "saneado" de relacionarse, propio de un mundo líquido en el que las identidades son fluidas (Bauman, 2005, 150).

En este mundo líquido las personas ya se relacionan tanto en el entorno offline como en el online; más aún, las relaciones sociales están ya plenamente hibridadas entre ambos contextos. Puesto que los medios digitales ofrecen una suerte de "remediación" (Bolter y Grusin, 2000) de las formas de comunicación previas, las mediaciones digitales son en realidad modos de volver a mediar interacciones, prácticas, formas de comunicación que ya estaban siendo mediadas (por el lenguaje, la escritura, los gestos y acentos, la apariencia personal, etc.). Sin embargo al mismo tiempo es evidente que, dada su condición electrónica, el ámbito digital presenta peculiaridades propias frente al medio presencial. Hoy en día la vida social tradicional, que es más lenta y localizada, coexiste con la vida social digital (más rápida y desarraigada). Son dos regímenes espacio-temporales y, aunque no es factible que el régimen tecnológico pueda algún día llegar a anular al tradicional -puesto que este es condición de posibilidad de aquel-; no cabe duda de que la coexistencia de ambos regímenes implica una mayor complejidad en las interacciones cotidianas. Así, la capacidad de los medios digitales para desdoblar la presencia de la persona, permitiéndole estar al mismo tiempo en dos lugares (el contexto en que se encuentra físicamente y el ámbito tecnológicamente mediado al que accede a través de las pantallas), es una fuente de desafíos y de posibles tensiones desde el punto de vista de la dimensión emocional de la persona (González, 2013).

En esta sociedad multipantalla, en la que la información circula prioritariamente a través de círculos sociales, la "cultura de la conectividad" imperante (Van Dijck, 2013) está configurando un nuevo marco de relación entre internet y las personas, pues para muchos -sobre todo jóvenes y hombres y mujeres de negocios- permanecer en línea se ha convertido en una necesidad social y laboral. Especialmente en relación con los dispositivos móviles, se han generado unas expectativas de uso ubicuas (Ling, 2014), como refleja el hecho de que "para la mayoría de los jóvenes (...) la posición de «apagado» ha sido borrada de su modelo cultural de teléfono móvil. Desde un punto de vista fenomenológico, simplemente no existe" (Caron y Caronia, 2007, 41). Este "contacto perpetuo" (Katz y Aakhus, 2002) no está exento de ciertas disonancias emocionales, ya que "cuando enviamos un SMS, esperamos que el destinatario esté «conectado» permanentemente y así, un retraso en la respuesta puede ser considerado como un desafecto personal. El teléfono móvil «nos libera» (para darnos movilidad) pero se convierte en una atadura, ya que se espera que estemos siempre localizables, siempre «de guardia»" (Hjorth, 2009, 129). 
Con todo ello, cabe afirmar que la conectividad permanente para los jóvenes no es solo un hábito, sino un componente esencial en su manera de construir y manejar sus amistades y su vida social. No obstante, como apuntan Hall y Baym (2012), un empleo demasiado intenso de las tecnologías digitales suscita con frecuencia en las personas una tensión entre el deseo de estar unidas a través de la tecnología y sentirse al mismo tiempo atrapadas por ella. Uno de los fenómenos que se derivan de ello es el descrito como "síndrome FOMO" (Fear Of Missing Out): el temor a estar perdiéndose algo lleva al usuario a la incapacidad de privarse de internet (Przybylski et al., 2013). Temor que también se refleja en el miedo de algunos usuarios de volverse invisibles frente a sus contactos, si dejan de actualizar su estado en las diferentes redes sociales. Estar conectado implica esencialmente estar visible, ya que "la visibilidad garantiza la inclusión en un mundo cuya representación se ha desplazado de lo palpable a lo comunicable (...) La clave que explica lo trascendente que se ha vuelto estar visible radica en lo amenazadora que resulta la invisibilidad. En términos de impacto social, para los jóvenes lo que no puede ser visto en los medios o subido a la red no existe" (Winocur, 2009, 69).

Así pues, en un medio -el digital-, marcado por la interconectividad y donde la persona no puede reafirmar su "yo" si no es visible para los demás, interactuar con otros a menudo no lleva aparejado un intercambio de información relevante, sino el desarrollo de una comunicación fática, para no perder el contacto social (Miller, 2008). En este sentido, siguiendo a Grusin, "dejar múltiples trazas de uno mismo en las redes sociales es visto como un objetivo necesario; e interactuar en dichas redes es placentero o deseable en parte porque estas trabajan para producir y mantener relaciones afectivas positivas con sus usuarios, para crear circuitos de feedback afectivos que hagan que uno quiera prodigarse en esas transacciones mediáticas" (2010, 4-5).

Por otra parte, una de las transformaciones más importantes que se derivan de la consolidación de las tecnologías digitales es la referente a la dimensión temporal. Las tecnologías digitales contribuyen a modelar -y al mismo tiempo son su mejor muestra- la cultura de la velocidad propia de nuestra época (Tomlinson, 2007; Poscente, 2008). Han provocado una aceleración sin precedentes de la percepción del tiempo (Wajcman, 2015), trayendo consigo cambios en los procesos de producción y consumo, la organización del trabajo, los estilos de vida o el modo en que el cerebro procesa la información (Small y Vorgan, 2009). Asistimos a una inflación del ahora, un "presentismo" (Rushkoff, 2013) que también influye en el tipo de consumo mediático, en el sentido de que puede conducir a sobrevalorar lo que ocurre a cada instante y a estar deseoso de lo nuevo, de lo inmediato. "Somos «neofílicos», hasta el extremo de superponer novedades triviales a informaciones más antiguas y valiosas", como afirma Reig $(2013,27)$.

Frente a todos los rasgos anteriormente descritos, y que configuran una "era de la hiperconectividad" (Reig y Vílchez, 2013), existe una corriente creciente de autores que alerta de los posibles perjuicios 
que la conexión digital permanente puede acarrear, ya sean de orden neurológico, afectivo o social. Así, en contraposición al "maximalismo digital" (Powers, 2010) -esto es, la creencia de que cuanto más tiempo esté uno conectado a las pantallas, mejor-; va consolidándose la convicción de que la desconexión digital comporta beneficios para la persona. Más aún, que su práctica habitual es un elemento necesario para la adquisición de la competencia digital.

La apuesta por la desconexión digital puede enmarcarse dentro de una corriente más amplia -el movimiento slow - que, en respuesta a la cultura de la velocidad antes citada, aboga por un estilo de vida más pausado. El movimiento slow denuncia la cultura de la prisa y sus consecuencias (la falta de paciencia, la hiperestimulación, la superficialidad y la multitarea, entre otras); frente a lo cual reivindica una forma alternativa de vida que cuestiona cualquier aceleración que no incorpore calidad a las diferentes actividades cotidianas (Honoré, 2004). Su premisa no es la defensa de la lentitud por la lentitud, sino la exigencia de conciliar el dinamismo propio de la sociedad de la información con un ritmo de vida adecuado para el hombre, en función de las características de sus acciones y de sus necesidades.

Por lo que atañe al uso de las tecnologías digitales, también comienza a teorizarse sobre la conveniencia de desarrollar una "comunicación slow" (Freeman, 2009; Rauch, 2011; Barranquero, 2013; SerranoPuche, 2014). Esta se basa en una revalorización del mundo físico y sus atributos frente al entorno online, con el objetivo de poner a la tecnología al servicio del hombre, para que este gane control sobre su vida y pueda aprovechar mejor los mensajes y contenidos tanto de sus comunicaciones digitales como presenciales. Por ello, el eje de la comunicación slow es la instauración de períodos regulares de desconexión digital para cultivar la comunicación cara a cara, abogando por el desarrollo de un ocio que prescinda de las tecnologías digitales y por una recuperación de las fronteras entre el tiempo laboral y el de descanso, que con la conectividad permanente quedan desdibujadas (Wajcman et al., 2009).

La defensa de la desconexión digital se apoya además en los beneficios que comporta para la recuperación de la capacidad de atención, pues navegar por internet exige una forma particularmente intensiva de multitarea mental, dado que la red es, por su mismo diseño, un sistema de interrupción y de fragmentación de la atención (Carr, 2011; Rosen, 2012). Como se ha demostrado, el multitasking merma la capacidad de pensar de manera profunda y creativa (Jackson, 2008). Además, gracias a la desconexión periódica se conseguiría que los momentos de conexión online fueran a su vez más enriquecedores, pues estar atado al flujo incesante de la información, paradójicamente, reduce la productividad y la eficacia.

Como veremos con más detalle en el siguiente epígrafe, en los últimos años ha ido aumentando la literatura académica y divulgativa que aborda la cuestión de la desconexión digital, tanto en 
investigaciones teóricas como empíricas. En ellas, con frecuencia se concluye que quienes practican el unplugging reconocen haber vivido en ese tiempo de desconexión una sensación de liberación y de paz, una mejor comunicación con sus familiares y amigos cercanos y la posibilidad de recuperar -más allá de las pantallas - tiempo para otras actividades necesarias y dignas de atención.

\section{El unplugging a estudio}

En mayo de 2013 el periodista norteamericano Paul Miller llamó la atención de la red con su artículo "I'm still here: back online after a year without the internet"1. En él relataba sus experiencias tras vivir un año desconectado de internet: desde el desconcierto inicial, al enfado o la incomprensión de algunos de sus amigos, a su redescubrimiento de algunos excesos y carencias en su modo de vida hiperconectado. En su relato explica como "hay mucho de realidad en lo virtual y mucho de virtual en nuestra realidad".

El fenómeno del unplugging como una práctica deseable e incluso necesaria para reencontrar un cierto equilibrio vital en una sociedad altamente tecnologizada está siendo objeto de múltiples estudios empíricos en los últimos años desde diversas ópticas. Muchos son los que se han preguntado por las causas que explican esa hiperconectividad, pensando que solo desde un diagnóstico adecuado se podrá afrontar cualquier propuesta de mejora. Este primer paso es interesante, ya que parece razonable pensar que el deseo de estar conectado de un modo contínuo debe tener alguna explicación que está más alla de la propia tecnología.

Una primera aportación es la de Humphreys (2005) que se pregunta por el rol del móvil en la normativa de las interacciones sociales. En su interesante investigación pone de manifiesto cómo el teléfono móvil reafirma la propuesta de Goffman $(1963,1971)$ sobre la existencia de "singles" y "withs" en los espacios públicos, y se convierte en un elemento que protege al "single", más vulnerable en estos entornos, de posibles invasiones o le ayuda a justificarse. Su propuesta teórica, fundamentada también con un estudio de campo, pone de manifiesto que el móvil ha adquirido en nuestras sociedades un papel clave para el individuo que transita de modo solitario por los espacios públicos: comer solo, viajar solo en el autobús, pasear solo por la calle, se justifica ante los demás si lo hacemos hablando por el móvil, o consultando algo en la pantalla, al tiempo que hace que el sujeto se sienta más protegido frente a una posible "agresión" externa. Esta perspectiva es especialmente interesante cuando se trata de educar en el buen uso del móvil y, ante cualquier intento de presentar el unplugging como una medida interesante merecería la pena tener en cuenta esta visión.

Además de este primer enfoque normativo, otros trabajos han profundizado de un modo empírico

1 http://www.theverge.com/2013/5/1/4279674/im-still-here-back-online-after-a-year-without-the-internet 
en las causas del deseo de estar conectados. Gonzales y Hancock (2011), por ejemplo, ponen de manifiesto que la información que los usuarios publican en sus perfiles en Facebook mejora su autoestima. La red social permite editar y controlar qué y cuándo se publica y comparte una determinada imagen o fotografía, lo que revierte positivamente en la propia consideración del sujeto. Gibbs (2006), McKenna et al. (2002), Valkenburg y Peter (2009), por su parte, coinciden en apuntar a la capacidad de la comunicación mediada por la tecnología (CMC, computer mediated communication) para generar una sensación de mayor intimidad que la comunicación cara a cara, lo que explica que el uso de internet se relacione de un modo consistente con un mayor bienestar social (Valenzuela et al., 2009).

Sin duda existen también otros estudios que ponen de relieve los aspectos menos positivos de esta hiperconectividad que parece potenciada de un modo particular por el binomio móviles y redes sociales. Chou y Edge (2011), por su parte, explican cómo los usuarios más habituales de Facebook, y aquellos que tienen entre sus contactos a numerosas personas que no conocen, tienden a sufrir sesgo de correspondencia o efecto de sobreatribución. Esto explica que los usuarios extienden una imagen o un estado compartido de uno de estos conocidos de baja intensidad (a quienes habitualmente no conocen en persona) y lo convierten en un atributo de su carácter: si alguien publica fotos o comentarios que expresan felicidad, se infiere de un modo simplista que esa persona es feliz.

Anderson y Rainie (2012) en un informe para Pew Research explican cómo los más jóvenes son quienes más se van a beneficiar pero también los más perjudicados por la hiperconectividad. De acuerdo con este estudio, el modo de vida actual les orienta a la gratificación instantánea, se acostumbran a las opciones rápidas y fomenta una clara impaciencia. Los expertos que participaron en este estudio señalaban como pertinente y necesaria una reforma de la educación con un mayor énfasis en la competencia digital.

En este contexto el estudio más relevante que vincula el unplugging con la educación de la competencia digital es "The World Unplugged", un proyecto desarrollado en 2010 que involucró a estudiantes de doce universidades en diez países del mundo. La propuesta metodológica del estudio era muy sencilla: los estudiantes que participaron en la investigación se abstuvieron de cualquier contacto con los medios y la tecnología durante 24 horas. Cada alumno podía elegir, dentro de un marco de tiempo determinado, cuándo se desconectaba. Durante la práctica, los participantes mantuvieron un diario donde anotaban las incidencias del experimento y al finalizar se realizaron también varios focus groups y sesiones de discusión con los participantes.

De acuerdo con Moeller, Powers y Roberts, coordinadores del estudio a nivel global, vivir la experiencia de estar desconectados permitió a los alumnos "ser más conscientes de la presencia de los medios de comunicación en sus vidas, tanto en beneficios como en limitaciones" $(2012$, 48). De un modo 
consistente, independientemente de su procedencia, todos los participantes señalaron un claro incremento de su conciencia sobre el papel de los medios. Este ayuno mediático les permitió también, en mayor o menor medida, identificar cierta adicción y dependencia de los medios y la tecnología, apreciar la incapacidad y el fracaso para intentar desconectarse, detectar cómo los móviles se han convertido en una extensión de uno mismo, ser conscientes del tiempo que se invierte en los medios, los beneficios que les reportan (principalmente mantener las relaciones cercanas) y algunos también experimentaron una cierta sensación de paz, libertad e incluso incremento de felicidad.

El experimiento tenía como único objetivo dejar de manifiesto que cualquier formación en la competencia mediática o digital pasa en primer término por ser consciente del papel que los medios y la tecnología juegan en la vida cotidiana. Y su propuesta metodológica es una dieta de 24 horas de unplugging. Los resultados confirman que la desconexión puede ayudar en el proceso de adquisición de la competencia digital, tal y como se propugna al inicio de este texto. El carácter del estudio, limitado tan solo a un día de modo puntual, no se vincula sin embargo con las propuestas de slow communication o de unplugging presentadas en el epígrafe anterior. Idóneamente, la correcta adquisición de la competencia digital llevaría consigo la determinación personal de encontrar algunos espacios de desconexión que permitan un uso crítico de la tecnología.

Y es que saber que la tecnología puede tener un efecto limitador no implica automáticamente un cambio en la conducta o un mayor nivel de autocontrol en su uso. En este sentido son interesantes los resultados obtenidos por Bringué y Sádaba en Iberoamérica (2010) y España (2009) cuando preguntan a los menores por su uso de la tecnología: mientras que el $72 \%$ de los menores en Latinoamérica y el 79\% en España pensaban que no pasaría nada si se quedarán dos semanas sin móvil, el $41 \%$ de los primeros y el 38\% de los segundos afirmaba no apagarlo nunca. Y en ese mismo sentido, solo un $7 \%$ de los menores iberoamericanos y un 3\% de los españoles afirmaba que su vida iría claramente a mejor si prescindieran del móvil durante quince días.

\section{Reflexiones finales}

En una sociedad cada vez más interconectada los ciudadanos necesitamos disponer de las capacidades que nos permitan estar a la altura de un nuevo mundo digital, no solo por medio de la adquisición de nuevas habilidades técnicas, sino a través de una mejor comprensión de las oportunidades, los desafíos e, incluso, las cuestiones éticas que plantean las nuevas tecnologías. Podemos decir que la educación mediática y digital es una cuestión clave en la formación de los futuros ciudadanos; pero, para realizarla de forma eficaz y obtener los resultados esperados, se requiere compaginar los aspectos técnicos, pedagógicos y los ético-cívicos del uso de los medios (Gonzálvez, 
2012), de modo que los ciudadanos adquieran los conocimientos, habilidades y competencias que les permitan comprender y usar adecuadamente dichas tecnologías.

Siguiendo las orientaciones de la UNESCO (2008), podemos señalar los aspectos y las competencias que conformarían la educación mediática e informacional. El acceso, evaluación y uso ético de la información; entender las funciones de los medios; la posibilidad de generar información; así como las capacidades de comprensión, pensamiento crítico, creatividad, conciencia intercultural y ciudadanía. Todos estos son aspectos que bien podrían conformar una propuesta práctica y educativa adecuada.

Tras analizar la literatura académica, divulgativa e investigaciones señaladas, consideramos que quizá convenga considerar la capacidad de desconexión como un componente sustancial de la competencia digital. No parece un objetivo sencillo de conseguir. Para lograr el hábito, al menos en parte, Sieberg (2011) articula un plan progresivo de cuatro pasos:

El primero consiste en examinar de modo crítico el consumo digital que uno realiza, para evaluar de qué modo el tiempo dedicado a la tecnología se ha perdido en detrimento de las relaciones familiares y sociales, de actividad física o de horas de sueño.

El segundo paso atañe propiamente a la fase de "desintoxicación" (digital detox): la abstinencia en el uso de la tecnología, de manera creciente (empezando quizá por algunas horas, después algún día o todo el fin de semana y retomando paralelamente tareas como la lectura, el deporte o las conversaciones cara a cara).

La tercera etapa es la de reconectarse digitalmente, pero partiendo ya de una serie de hábitos saludables -entre otros, fijar de manera estable y periódica los momentos de desconexión- y reasignando a la tecnología el lugar que le ha de corresponder dentro del conjunto de actividades diarias.

Todo ello, por último, conduce al desarrollo de una concepción renovada de la comunicación y de la vida digital, de manera que la relación con la tecnología y la relación presencial con las personas fluyan de modo natural y equilibrado.

En definitiva y como apunta Reig, los ciudadanos de la sociedad de la información debemos saber aprovechar las ventajas de ambas situaciones (estar online y estar offline), es decir, "alternar momentos de conectividad, colaboración y cocreación, con otros de concentración y creatividad individual (...) Pasar de la felicidad basada en el reconocimiento social a la que disfruta de la autorrealización, evolucionar hacia hacernos más independientes de la tiranía de la deseabilidad social” $(2013,46)$.

Esto requiere enseñar a los jóvenes a prescindir de la sobrecarga de contenidos vacíos y a reducir la dependencia tecnológica, invirtiendo parte de ese tiempo en cultivar otras relaciones o aficiones, a la 
vez que aprenden a aprovechar los momentos de conexión con mayor idoneidad (Aguaded y RomeroRodríguez, 2015). Lograr este objetivo implica, no lo dudamos, contar con familias sensibilizadas, profesores preparados, profesionales de los medios de comunicación responsables y autoridades políticas comprometidas. Del mismo modo, parece conveniente seguir indagando, en la línea que ya se ha iniciado, sobre la eficacia de la media literacy education, tratando de individuar factores que ayuden a conseguirlo, tales como: valorar el impacto de los métodos de instrucción; explorar el papel que juegan las diferencias individuales o la influencia de los contextos sociales, entre otros.

\section{Referencias}

Aguaded, I. y Romero-Rodríguez, L. M. (2015). Mediamorfosis y desinformación en la infoesfera: alfabetización mediática, digital e informacional ante los cambios de hábitos de consumo informativo. Education inthe KnowledgeSociety(EKS), 16(1), 44-57. doi:http://dx.doi.org/10.14201/eks20151614457

Anderson, J. Q. y Rainie, L.(2012). Millennials will benefit and suffer due to their hyperconnected lives. En The future of the Internet Series, Pew Research Center, Washington DC, http://www.pewinternet. org/Reports/2012/Hyperconnected-lives/Oveview.aspx. Recuperado el 24 de abril de 2015.

Barranquero, A. (2013). Slow media. Comunicación, cambio social y sostenibilidad en la era del torrente mediático. Palabra Clave, 16(2), 419-448.

Bauman, Z. (2005). Identidad: conversaciones con Benedetto Vecchi. Madrid: Losada.

Bawden, D. (2008). Origins and concepts of digital literacy. En C. Lankshear y M. Knobel (Eds.), Digital literacies: concepts, policies and practices. New York: Peter Lang.

Bolter, J. D. y Grusin, R. (2000). Remediation: Understanding New Media. Cambridge (MA): The MIT Press.

Bringué, X. y Sádaba, Ch. (2009). La Generación Interactiva en España: jóvenes y adolescentes frente a las pantallas. Barcelona: Ariel.

Bringué, X., Sádaba, Ch. y Tolsá, J. (2010). La Generación Interactiva en Iberoamérica: jóvenes y adolescentes ante las pantallas. Madrid: Foro Generaciones Interactivas-Fundación Telefónica.

Bundy, A. (Ed.) (2004). Australian and New Zealand information leteracy framework, principles, standards and practice. Adelaide: Australian and New Zealand Institute for Information Literacy.

Cáceres, M. D. y Morales, E. (2014). Juventud hiperconectada. Comunicación y sociabilidad virtual. Anuario electrónico de estudios en comunicación social, 7(2), 160-177. 
Campuzano, A. (1992). Tecnologías audiovisuales y educación. Una visión desde la práctica. Madrid: Akal.

Caron, A. H. y Caronia, L. (2007). Moving Cultures: Mobile Communication in Everyday Life. Montreal: McGill-Queen's University Press.

Carr, N. (2011). Superficiales: ¿qué está haciendo Internet con nuestras mentes? Madrid: Taurus.

Chou, H-T. G., y Edge, N. (2012). They Are Happier and Having Better Lives than I Am: The Impact of Using Facebook on Perceptions of Others Lives. Cyberpsychology, Behavior, and Social Networking, 15(2), 117-121. doi: http://dx.doi.org/ 10.1089/cyber.2011.0324

Comisión Europea (2007). Competencias clave para el aprendizaje permanente. Un marco de referencia europeo. Luxemburgo: Oficina de Publicaciones Oficiales de las Comunidades Europeas. Recuperado el 13 de agosto de 2013 de: http://ec.europa.eu/dgs/education_culture/publ/ pdf/Ilearning/keycomp_es.pdf

Freeman, J. (2009). Manifesto for a Slow Communication Movement. En J. Freeman, The Tyranny of E-mail: The Four-Thousand-Year Journey to Your Inbox (pp. 190-203). New York: Scribner.

Gallado, E. E. (2013). Competencia digital: revisión integradora de la literatura. Revista de Ciencias de la Educación Academicus, 1(3), 56-62.

Gibbs J. L. (2006). Self-presentation in online personals: the role of anticipated future interaction, self-disclosure, and perceived success in Internet dating. Communication Research, 33, 152-177. doi: http://dx.doi.org/10.1177/0093650205285368

Gilster, P. (1997). Digital literacy. New York: Wiley.

Goffman, E. (1963). Behavior in Public Places: Notes on the Social Organization of Gatherings. New York: Free Press.

Goffman, E. (1971). Relations in Public: Microstudies of the Public Order. New York: Harper \& Row.

Gonzales, A. L. y Hancock, J. T. (2011). Mirror, Mirror on my Facebook Wall: Effects of Exposure to Facebook on Self-Esteem. Cyberpsychology, Behavior, and Social Networking, 14(1-2), 79-83. doi: http:// dx.doi.org/10.1089/cyber.2009.0411

González, A. M. (2013). Introducción: emociones y análisis social. En L. Flamarique y M. D’OliveiraMartins (Eds.), Emociones y estilos de vida: radiografía de nuestro tiempo (pp. 9-24). Madrid: Biblioteca Nueva. 
Gonzálvez, V. (2012). Ciudadanía mediática. Una mirada educativa. Madrid: Dykinson.

Grusin, R. (2010): Premediation: Affect and Mediality after 9/11. Basingstoke: Palgrave Macmillan. doi: http://dx.doi.org/10.1057/9780230275270

Hall, J. A. y Baym, N. K. (2012). Calling and texting (too much): Mobile maintenance expectations, (over) dependence, entrapment, and friendship satisfaction. New Media \& Society, 14(2), 316-331. doi: http://dx.doi.org/10.1177/1461444811415047

Hawkins, R. \& Paris, A. E. (1997). Computer literacy and computer use among college students: differences in blackandWhite. Journalof Negro Education,66(2). doi:http://dx.doi.org/10.2307/2967224 Hjorth, L. (2009). La poética del retraso: medios móviles, tecnologías omnipresentes y nociones de lugar. En J. M. Aguado y I. J. Martínez (Eds.), Sociedad móvil: tecnología, identidad y cultura (pp. 119135). Madrid: Biblioteca Nueva.

Honoré, C. (2004). In praise of slowness. New York: Harper Collins.

Humphreys, L. (2005). Cellphones in public: social interactions in a wireless era. New media \& Society, 7(6), 810-833. doi:http://dx.doi.org/10.1177/1461444805058164.

Jackson, M. (2008). Distracted: The Erosion of Attention and the Coming DarkAge. New York: Prometheus. Jordan, T. (2013). Internet, Society and Culture. Communicative Practices Before and After the Internet. New York-London: Bloomsbury.

Katz, J. E. y Aakhus, M. (2002) (Eds.). Perpetual contact: Mobile communication, private talk, public performance. Cambridge: Cambridge University Press.

Kubey, R. (2003). Why US media education lags behind the rest of the English-speaking world. Television New Media, 4(4), 351-370. doi: http://dx.doi.org/10.1177/1527476403255808

Lasén, A. (2014). 'Introducción. Las mediaciones digitales de la educación sentimental de los y las jóvenes'. En I. Megía Quirós y E. Rodríguez San Julián, Jóvenes y Comunicación. La impronta de lo virtual (pp. 7-16). Madrid: Fundación de Ayuda contra la Drogadicción.

Ling, R. (2014). From ubicomp to ubiex (pectations). Telematics and Informatics, 31(2), 173-183. doi: http://dx.doi.org/10.1016/j.tele.2013.09.001

Lipovetsky, G. (2006). Los tiempos hipermodernos. Barcelona: Anagrama.

Martens, H. (2010). Evaluating Media Literacy Education. Concepts, Theories, and Future Directions. Journal of Media Literacy Education, 2(1), 1-22. 
McKenna KYA, Green AS, Gleason M. (2002). Relationship formation on the Internet: what's the big attraction? Journal of Social Issues, 58, 9-31.

Miller, D. (2008). New Media, Networking and Phatic Culture. Convergence: The International Journal of ResearchintoNewMediaTechnologies, 14(4),387-400.doi:http://dx.doi.org/10.1177/1354856508094659

Moeller, S., Powers, E. y Roberts, J. (2012). El mundo desconectado y 24 horas sin medios: alfabetización mediática para la conciencia crítica de los jóvenes. Comunicar, 20(39), 45-52. doi: http:// dx.doi.org/10.3916/C39-2012-02-04

Naval, C. y Arbués, E. (2012). La alfabetización mediática de los futuros profesores de educación primaria y secundaria. En L. García Aretio (Ed.), Sociedad del conocimiento y educación (pp. 93-102). Madrid: Universidad Nacional de Educación a Distancia.

Naval y Arbués (2013). Training in media and audiovisual competence. 7th Annual Conference Democratic Citizenship and Human Rights Education. Birbeck, University of London and Institute of Education (28 de junio de 2013).

Naval, C. y Arbués, E. (2014). La alfabetización mediática de los futuros profesores de educación primaria y secundaria en un entorno intercultural. En F. Stara, La costruzione del pensiero e delle strategie interculturali (pp. 69-91). Lecce: Pensa Multimedia.

Papacharissi, Z. (Ed.) (2011). A Networked Self: Identity, Community, and Culture on Social Network Sites. New York: Routledge.

Parlamento Europeo. Informe sobre la alfabetización de los medios de comunicación en un mundo digital (2008/2129(INI)). Recuperado el 20 de junio de 2013 de: http://www.europarl.europa.eu/sides/ getDoc.do?pubRef=-//EP//TEXT+REPORT +A6-2008-0461+0+DOC+XML+V0//ES

Pavón, F. (2005). Educación para las nuevas tecnologías. Pixel-Bit. Revista de Medios y Educación, 25, $5-17$.

Pérez-Tornero, J. M. y Martínez Cerdá, J. F. (2011). Hacia un sistema supranacional de indicadores mediáticos. Infoamérica ICR, 5, 39-57.

Pisani, F. y Piotet, D. (2009). La alquimia de las multitudes: cómo la web está cambiando el mundo. Barcelona: Paidós.

Poscente, V. (2008). The age of speed: learning to thrive in a more-faster-now world. New York: Ballantine. Potter, W. J. (2010). The state of media literacy. Journal of Broadcasting \& Electronic Media, 54(4), 675696. doi:http://dx.doi.org/10.1080/08838151.2011.521462 
Powers, W. (2010). Hamlet's Blackberry. A practical philosophy for building a good life in the digital age. New York: Harper Collins.

Przybylski, A. K., Murayama, K., Dehaan, C. R. y Gladwell, V. (2013). Motivational, emotional, and behavioral correlates of fear of missing out. Computers in Human Behavior, 29,1841-1848. doi:http:// dx.doi.org/10.1016/j.chb.2013.02.014

Rainie, L. y Wellman, B. (2012). Networked. The New Social Operating System. Cambridge, MA: The MIT Press.

Rauch, J. (2011). The Origin of Slow Media: Early diffusion of a cultural innovation through popular and press discourse, 2002-2010. Transformations: Journal of Media \& Culture, 20.

Reig, D. (2013). Describiendo al hiperindividuo, el nuevo individuo conectado. En D. Reig y L. Vílchez, Losjóvenes en la era de la hiperconectividad: tendencias, claves y miradas (pp. 23-90). Madrid: Fundación Telefónica.

Reig, D. y Vílchez, L (2013). Los jóvenes en la era de la hiperconectividad: tendencias, claves y miradas. Madrid: Fundación Telefónica.

Rosen, L. (2012). iDisorder: Understanding Our Obsession with Technology and Overcoming Its Hold on Us. Basingstoke: Palgrave Macmillan.

Rosenbaum, J. E., Beentjes, J. W. J. and Konig, R. P. (2008). Mapping media literacy: Key concepts and future directions. En C. S. Beck (Ed.), Communication Yearbook 32 (pp. 313-353). New York: Routledge. Rushkoff, D. (2013). Present Shock. When Everything Happens Now. New York: The Penguin Group.

Sancho, J. M. (2001). Repensando el significado y metas de la educación en la sociedad de la información. El efecto fractal. En M. Ara (Coord), Educar en la sociedad de la información (pp. 37-79). Bilbao: Desclée.

Serrano-Puche, J. (2014). Hacia una comunicación slow: el hábito de la desconexión digital periódica como elemento de alfabetización mediática. Trípodos, 34, 201-214.

Sieberg, D. (2011). Digital Diet: The 4-Step Plan to Break Your Addiction and Regain Balance in Your Life. New York: Three River Press.

Small, G. y Vorgan, G. (2009). iBrain: Surviving the Technological Alteration of the Modern Mind. New York: Harper Collins.

Tomlinson, J. (2007). The culture of speed: the coming of immediacy. Los Angeles: Sage. 
Turkle, S. (2011). Alone together. Why We Expect More from Technology and Less from Each Other. New York: Basic Books.

UNESCO (2008). Teacher Training Curricula for Media and information Literacy. Report of the International Expert Group Meeting. Paris: UNESCO. Recuperado el 11 de junio de 2013 de: http:// portal.unesco.org/ci/fr/files/27508/12212271723Teacher-Training_Curriculum_for_MIL___final_ report.doc/TeacherTraining\%2B Curriculum\%2Bfor\%2BMIL\%2B-\%2Bfinal\%2Breport.doc.

Valenzuela S, Park N, y Kee K. (2009). Is there social capital in a social network site? Facebook use and college students' life satisfaction, trust, and participation. Journal of Computer-Mediated Communication, 14, 875-901. doi:http://dx.doi.org/10.1111/j.1083-6101.2009.01474.x

Valkenburg P. M. y Peter J. (2009). Social consequences of the Internet for adolescents: a decade of research. Current Directions in Psychological Science, 18, 1-5. doi:http://dx.doi.org/10.1111/j.14678721.2009.01595.x

Van Dijck, J. (2013). The Culture of Connectivity: A Critical History of Social Media. Oxford: Oxford University Press. doi:http://dx.doi.org/10.1093/acprof:oso/9780199970773.001.0001

Wajcman, J. (2015). Pressed for Time. The Acceleration of Life in Digital Capitalism. Chicago: The University of Chicago Press.

Wajcman, J., Bittman, M. y Brown, J. (2009). Intimate Connections: The Impact of the Mobile Phone on Work/Life Boundaries. En G. Goggin y L. Hjorth (Eds.), Mobile Technologies: From Telecommunications to Media (pp. 9-22). Nueva York: Routledge,

Wellman, B., Quan-Haase, A., Boase, J., Chen, W., Hampton, K., Díaz De Isla, I. y Miyata, K. (2003). The social affordances of the Internet for networked individualism. Journal of Computer-Mediated Communication, 8(3). doi:http://dx.doi.org/10.1111/j.1083-6101.2003.tb00216.x

Wilson, C., Grizzle, A., Tuazon, R., Akyempong, K. and Cheung, C. (2011). Media and Information literacy curriculum for teachers. Paris: UNESCO.

Winocur, R. (2009). Robison Crusoe ya tiene celular. México: Siglo XXI Editores. 